\title{
The influence of interactions and minor mergers on the structure of galactic disks ${ }^{\star}$
}

\author{
I. Observations and disk models \\ U. Schwarzkopf $f^{1,2, \star \star}$ and R.-J. Dettmar ${ }^{1}$ \\ 1 Astronomisches Institut, Ruhr-Universität Bochum, Universitätsstraße 150, 44780 Bochum, Germany \\ 2 Steward Observatory, University of Arizona, 933 N. Cherry Ave., Tucson, Arizona 85721, U.S.A.
}

Received July 22, 1999; accepted March 6, 2000

\begin{abstract}
This paper is the first part in our series on the influence of tidal interactions and minor mergers on the radial and vertical disk structure of spiral galaxies. We report on the sample selection, our observations, and data reduction. Surface photometry of the optical and near infrared data of a sample of 110 highly-inclined/edge-on disk galaxies are presented. This sample consists of two subsamples of 61 non-interacting galaxies (control sample) and of 49 interacting galaxies/minor merging candidates. Additionally, 41 of these galaxies were observed in the near infrared. We show that the distribution of morphological types of both subsamples is almost indistinguishable, covering the range between $0 \leq T \leq 9$. An improved, 3-dimensional disk modelling- and fitting procedure is described in order to analyze and to compare the disk structure of our sample galaxies by using characteristic parameters. We find that the vertical brightness profiles of galactic disks respond very sensitive even to small deviations from the perfect edge-on orientation. Hence, projection effects of slightly inclined disks may cause substantial changes in the value of the disk scale height and must therefore be considered in the subsequent study.
\end{abstract}

Key words: galaxies: general, interactions, photometry, spiral, statistics — infrared: galaxies

Send offprint requests to: U. Schwarzkopf,

e-mail: schwarz@as.arizona.edu

* Based on observations obtained at the European Southern Observatory (ESO, La Silla, Chile), Calar Alto Observatory operated by the MPIA (DSAZ, Spain), Lowell Observatory (Flagstaff/AZ, USA), and Hoher List Observatory (Germany).

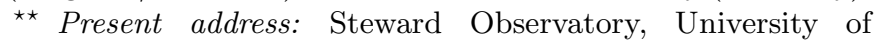
Arizona, 933 N. Cherry Ave., Tucson, Arizona 85721, U.S.A.

\section{Introduction}

Considering the fact that the majority of (spiral) galaxies is not completely isolated but located in an environment which enables repeated close encounters or even merging processes with small companions it seems to be meaningful to systematically investigate the properties of galaxies affected by such processes. The investigation of their structural and dynamical changes caused by tidal interactions or low-mass satellite infall - hence "minor merger" - can help to clarify how far the evolution of disk galaxies was modified or even dominated by environmental effects.

Several N-body simulations were performed during the last decade in order to study the influence of minor mergers on galactic disks in greater detail (e.g. Quinn et al. 1993; Mihos et al. 1995; Walker et al. 1996). It was possible to use more realistic, multiple-component models for the galaxy-satellite system - usually consisting of disk, bulge, and halo - as well as a large number of particles $\left(n_{\text {disk }} \geq 32000\right)$. One of the main conclusions was that even merging processes in the range between $M_{\text {sat }} / M_{\text {disk }} \approx 0.05-0.2$ can cause a vertical thickening of the stellar disk component by a factor between 2 and 4 , depending on the galactocentric distance. It was found that this vertical heating is due to a gain of kinetic energy of the disk stars by enhanced two-body relaxation. According to a series of papers on the frequency of these so called "soft merging" events (e.g. Toth \& Ostriker 1992; Zaritsky 1995, 1996) a large number of present-day (disk-) galaxies were affected by merging- or accretion processes of this magnitude since they have formed. As a consequence, interactions and minor mergers within this mass range might modify our picture of galaxy formation and evolution.

However, the enormous parameter space of such a complex scenario makes it difficult to derive general conclusions from a set of few specific simulations. 
The quantitative results still crucially depend on the chosen parameters such as the content and behaviour of gas in the disk, the mass ratio between bulge and disk, induced star formation, or the satellite orbit (Quinn et al. 1993; Mihos et al. 1995; Velazquez \& White 1999).

Statistical studies of galaxy interactions - based on optical photometry of disk galaxies (Reshetnikov \& Combes 1996, 1997) - focused on the effects of tidally-triggered disk thickening between systems of comparable mass. They found that the ratio $h / z_{0}$ of the radial exponential scale length $h$ to the constant scale height $z_{0}$ is only about twice smaller for interacting galaxies - a lower value than derived from the minor mergers simulations. However, the small number of objects in their sample (7 non-interacting and 24 interacting galaxies) did not permit to study these questions in detail.

Therefore, we started a project based on a larger sample of edge-on disk galaxies in both optical and near infrared passbands. This combination offers a number of advantages:

First, observations in the near infrared particularly benefit from the much lower dust extinction near the galactic plane, i.e. at small $z$. Second, the presence of a dust lane along the major axis of most edge-on disk galaxies still presents one of the best methods to determine precise inclinations of the disks - two facts that will become very important in order to derive reliable scale parameters from a disk fitting procedure. Third, this combination enables us to make conclusions on disk populations of different ages.

The main questions of this study can be summarized as follows:

- Are interactions/minor mergers able to change the radial and vertical structure of affected galactic disks?

- Is there a substantial vertical disk thickening?

- Of which order are the differences and similarities in the disk parameter distribution for a sample of interacting/non-interacting galaxies, respectively?

- To what extent are the disk properties of galaxies in the local universe influenced by interactions/minor mergers?

Due to the complexity of these questions the paper is split into three parts: in this first part (Paper I) we present deep optical and near infrared photometric data of a total sample of 110 highly-inclined/edge-on disk galaxies. This sample consists of two subsamples of 61 non-interacting galaxies (control sample) and of 49 minor merging candidates. Additionally, 41 of these galaxies were observed in the near infrared. In Sect. 2 the criteria of the sample selection will be described briefly. Section 3 gives an overview on the observations and data reduction. The disk modelling- and fitting procedure applied to derive the disk parameters will be reviewed in Sect. 4 . In Sect. 5 we summarize and conclude the paper.
In the second part (Schwarzkopf \& Dettmar 2000, Paper II) the results of a detailed analysis of the structure of galactic disks will be presented.

The third part (Schwarzkopf \& Dettmar in preparation, Paper III) will be focused on the influence of accompanying minor merger features - like disk "warping" and "flaring" - on the vertical disk structure.

\section{Sample selection}

\subsection{General remarks}

The selection of two separate subsamples of highlyinclined disk galaxies - consisting of a large number of interacting/merging candidates in the mass range $M_{\text {sat }} / M_{\text {disk }} \approx 0.1$, and of relatively isolated disk galaxies - is a crucial point for the comparison of disk parameters. Since presumably not all of the merging processes of this order of magnitude - compared to large merging events are able to change the structure of affected galaxy disks completely, two facts must be considered in order to classify both subsamples:

First, only merging events in a more progressed phase will have any appreciable effect on the disk structure. We therefore did not consider candidates in an early stage of interaction. Instead, the sample contains some highlyinclined disk galaxies that show no separate satellite merging with the disk component, but strong evidence for accretion in the recent past (indicated by both a disturbed disk structure and characteristic effects such as warping and tidal tails). Most of these objects are located in galaxy groups with a few members, e.g. NGC 3628, NGC 4634, or NGC 4762. Due to their perturbed structure a morphological classification is difficult.

Second, a control sample of non-interacting edge-on disk galaxies must include all morphological types in the range $0 \leq T \leq 9$ (Table 1 ). The galaxies should be isolated without indication of interaction and accretion. In particular, it is important to include the latest type spirals like Sd - so called "superthin" galaxies - since they belong to a class of disk galaxies having the smallest known axis ratios (between 1:9 and 1:20). These galaxies are characterized by velocity curves of modest gradient (Goad \& Roberts 1981; Griv \& Peter 1996a-c), indicating that the "superthins" represent kinematically almost unheated galaxy disks.

Beyond these "limitations" and the specific criteria described in the next section it seems difficult to give a generalized definition of the selection criteria. As a consequence, it is unavoidable that the sample of non-interacting galaxies is slightly "polluted" by some galaxies that were affected by interactions/minor mergers during their past but show no indication of these effects today. This effect would, however, only influence the non-interacting galaxies and lead to an underestimation of the actual differences between both samples. 
Table 1. Nomenclature of morphological galaxy types (cross reference). Columns: (1) Source: ESO-LV = Lauberts \& Valentijn (1989), Hubble = Hubble (1926), ESO-Upp = "old" type in Lauberts \& Valentijn (1989); (2) Designation of morphological types

\begin{tabular}{|c|c|c|c|c|c|c|c|c|c|c|c|c|c|c|c|c|}
\hline \multirow{2}{*}{$\begin{array}{l}\text { Source } \\
\qquad(1) \\
\text { ESO-LV }\end{array}$} & \multicolumn{16}{|c|}{$\begin{array}{c}\text { Morphological Type } \\
(2)\end{array}$} \\
\hline & -5 & -4 & -3 & -2 & -1 & 0 & 1 & 2 & 3 & 4 & 5 & 6 & 7 & 8 & 10 & 11 \\
\hline Hubble & $\mathrm{E}$ & - & E-S0 & S0 & - & $\mathrm{S} 0 / \mathrm{a}$ & $\mathrm{Sa}$ & Sa-b & $\mathrm{Sb}$ & $\mathrm{Sb}-\mathrm{c}$ & - & Sc & - & Sc-Irr & $-\quad \operatorname{IrrI}$ & - \\
\hline ESO-Upp & $\mathrm{E}$ & - & E-S0 & S0 & - & S0-a & $\mathrm{Sa}$ & Sa-b & $\mathrm{Sb}$ & Sb-c & S... & $\mathrm{Sc}, \mathrm{Sc}-\mathrm{d}$ & S.../Irr & $\mathrm{Sd}$ & Irr & unknown \\
\hline
\end{tabular}
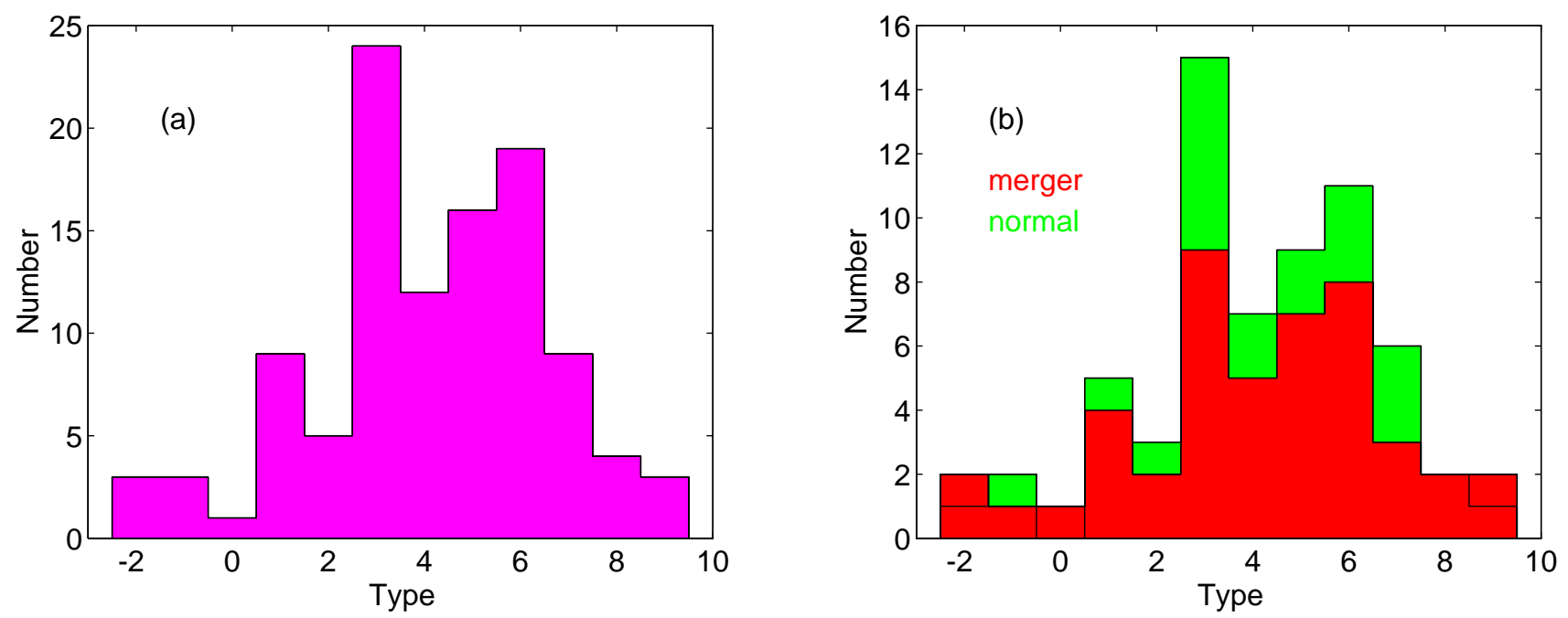

Fig. 1a and $\mathbf{b}$. The distribution of morphological types a) for the total galaxy sample and $\mathbf{b}$ ) for both subsamples of non-interacting (normal) and interacting/merging galaxies (merger)

\subsection{The sample of interacting/merging galaxies}

In this study, the notation "interacting/merging" refers closely to the classification scheme introduced by Arp \& Madore (1987) and will therefore be used as a synonym for all galaxies which fulfil the following criteria:

Galaxies with interacting companions, interacting doubles, galaxies with peculiar disks, galaxies with tails, loops of material or debris, irregular or disturbed galaxies, chains and groups of galaxies. A more detailed description of these classifications is given in Arp \& Madore (1987).

With these limitations in mind, we selected interacting/merging candidates with an inclination $i \geq 85^{\circ}$ (derived from a first visual inspection). During this preselection we did not impose any morphological restrictions except that their type should be not much earlier than $T \approx 0$. The candidates were chosen from optical prints in "A Catalogue of Southern Peculiar Galaxies and Associations" (Arp \& Madore 1987), "Atlas of Peculiar Galaxies" (Arp 1966), and the NASA "Atlas of Galaxies" (Sandage \& Bedke 1988). We also selected some systems from the catalogue "Satellites of Spiral Galaxies" (Zaritsky et al. 1993, 1997).
Another selection criterion for the minor mergers and those galaxies in the subsample with very close companions was the mass ratio between the main bodies. For all the candidates which could be separated into two individuals (i.e. for $\approx 20$ galaxies of the interacting/merging sample) this ratio was checked by an estimation of their total fluxes within a certain aperture or ellipse that contains all intensities down to the sky brightness. The resulting mean ratio is $M_{\text {sat }} / M_{\text {disk }} \approx 0.08 \pm 0.035$. Typical examples (with errors of $\approx 0.005)$ are NGC $1531 / 32(\approx 0.05)$, NGC $128(\approx 0.045)$ or NGC $1888(\approx 0.07)$. For interacting galaxies located a less dense group or for those with a remote companion this mass ratio can be larger.

\subsection{The sample of non-interacting galaxies}

The selection criteria for disk inclination and morphological types were the same as described for the interacting/merging galaxies. Our principal sources for the subsample of highly-inclined, non-interacting galaxies were the ESO-Uppsala catalogue (Lauberts \& Valentijn 1989), the RC3- (de Vaucouleurs et al. 1991) and UGC (Nilson 1973) catalogues, the "Carnegie Atlas of Galaxies" (Sandage \& Bedke 1994), and "The 
Hubble Atlas of Galaxies" (Sandage 1961). To check their isolation, larger fields $\left(\approx 10^{\prime}-15^{\prime}\right.$, depending on the distance of individual objects) were inspected visually using the Digitized Sky Survey ${ }^{1}$.

In order to benefit from a better spatial resolution of some closer objects there was only a lower limit in apparent angular disk diameter of $\geq 2^{\prime}$ for both galaxy samples. Finally, the sample of non-interacting galaxies was filled up by 11 edge-on galaxies of the Barteldrees \& Dettmar (1994) data set.

\subsection{Distribution of morphological types}

Several studies of the properties of edge-on galaxies argued that some of the disk parameters of spiral galaxies, e.g. the ratio of disk scale length to scale height, might be correlated with the morphological type (de Grijs \& van der Kruit 1996; de Grijs 1997; Schwarzkopf \& Dettmar 1998). Therefore, it was also important to ensure that both subsamples investigated in this study are not affected by selection effects. This applies, in particular, to the distribution of morphologigal types and to the redshifts/distances of selected objects. Due to their relation to the absolute properties of galactic disks the latter will be discussed in detail in Paper II.

The distribution of morphological types (according to $\mathrm{NED}^{2}$ ) is shown in Fig. 1a for the complete galaxy sample, and in Fig. 1b for both subsamples of non-interacting and interacting/merging galaxies, respectively. The statistical test of Kolmogorov \& Smirnov (Darling 1957; Sachs 1992) - hereafter KS - quantifies the similarity of both subsamples with a result of 0.04 . That is significantly lower than the value necessary for the $20 \%$-limit (0.2), which is the strongest of the KS-criteria. Considering also the unavoidable errors $(\Delta T \approx \pm 1)$ introduced by an automated type classification (Corwin et al. 1985; Lauberts \& Valentijn 1989) both distributions are statistically indistinguishable. The gross of galaxies covers the range between $3 \leq T \leq 7$, with a strong peak at $T=3$. This peak can also be observed in the frequency distribution of galaxy types in the available catalogues. The effect is caused by the selection criteria of the classification programs used in the catalogues (Sect. 6 in Lauberts \& Valentijn 1989).

\footnotetext{
1 The Digitized Sky Survey was produced at the Space Telescope Science Institute under U.S. Government grant NAG $\mathrm{W}-2166$.

2 The NASA/IPAC Extragalactic Database (NED) is operated by the Jet Propulsion Laboratory, California Institute of Technology, under contract with the National Aeronautics and Space Administration.
}

\section{Observations and data reduction}

\subsection{Optical observations}

Due to the large number of galaxies needed for reliable statistics the optical observations were obtained with different telescopes and during several observing runs between February 1996 and June 1998. The following telescopes were used:

$1.54 \mathrm{~m}$ Danish and $61 \mathrm{~cm}$ Bochum telescope on La Silla, Chile; 42-inch telescope at Lowell Observatory, Flagstaff; $1.23 \mathrm{~m}$ telescope at Calar Alto Observatory, Spain; $1.06 \mathrm{~m}$ telescope at Hoher List Observatory, Germany. The used passbands were Johnson $R$ and Thuan \& Gunn $r$, with a central wavelength at $\lambda_{\mathrm{c}}=652$ $\mathrm{nm}$ and $670 \mathrm{~nm}$, bandpass $\Delta \lambda=162 \mathrm{~nm}$ and $103 \mathrm{~nm}$, respectively.

All observing runs and the used telescope/detector characteristics are listed in Table 2 . The seeing conditions are given as averaged $F W H M$ values.

Details of the optical observations are listed in Table 4. Additionally for most of the sample galaxies the total blue surface brightness, $B_{\mathrm{T}}$, and the morphological type $T$ (revised Hubble type, according to Lauberts \& Valentijn 1989, Table 1) are given.

\subsection{Near infrared observations}

The near infrared observations were obtained with the MAGIC camera of the MPIA attached to the $2.2 \mathrm{~m}$ and $1.23 \mathrm{~m}$ telescopes of the Calar Alto Observatory, Spain, and with the IRAC2b camera on the ESO/MPIA $2.2 \mathrm{~m}$ telescope of the European Southern Observatory (ESO), Chile, respectively. Both the MAGIC and IRAC2b cameras are equipped with a Rockwell $256 \times 256$ pixel $^{2}$ NICMOS3 $\mathrm{HgCdTe}$ array. The data were acquired during several observing runs between February 1996 and June 1998.

For all runs we used the $K$ filter (central wavelength $\lambda_{\mathrm{c}}=2.20 \mu \mathrm{m}$, bandpass $\left.\Delta \lambda=0.40 \mu \mathrm{m}\right)$, or the $K^{\prime}$ filter $\left(\lambda_{\mathrm{c}}=2.15 \mu \mathrm{m}, \Delta \lambda=0.32 \mu \mathrm{m}\right)$. For some objects images in the $H$ filter were obtained $\left(\lambda_{\mathrm{c}}=1.65 \mu \mathrm{m}, \Delta \lambda=\right.$ $0.30 \mu \mathrm{m})$.

We recorded object and sky frames alternately, with typical single integration times of $10 \times 5$ s per sequence, and spatially separated by $\approx 6^{\prime}$. To eliminate bad pixels, the telescope "on source"-positions were dithered by a few arcseconds between subsequent exposures. Since many of the sample galaxies are faint objects it was required to reach at least $30-40$ min integration time "on source", which corresponds to $\approx 9-12$ cycle repetitions and a resulting observing time of $\approx 2.5-3$ hours. Therefore, only one third of our total optical galaxy sample was observed in the near infrared.

Since our study is aiming at an investigation of the structure and geometrical properties of galactic disks, 
Table 2. Observing dates and telescope/detector characteristics, given for optical and near infrared observations. Columns: (1) Observing dates; (2) Telescopes used: $2.2 \mathrm{CA}=2.2 \mathrm{~m}$ Calar Alto, 2.2 ESO $=2.2 \mathrm{~m} \mathrm{ESO} / \mathrm{MPIA}, 1.5 \mathrm{DA}=1.54 \mathrm{~m}$ Danish, 1.2 $\mathrm{CA}=1.23 \mathrm{~m}$ Calar Alto, $1.1 \mathrm{LO}=1.1 \mathrm{~m}$ Lowell, $1.0 \mathrm{HL}$ $=1.06 \mathrm{~m}$ Hoher List, 0.6 BO $=0.6 \mathrm{~m}$ Bochum; (3) Field of view; (4) Pixel scale; (5) Detector used; (6) Average seeing conditions $(F W H M)$

\begin{tabular}{|c|c|c|c|c|c|}
\hline $\begin{array}{c}\text { Observing } \\
\text { dates } \\
(1)\end{array}$ & Telescope & $\begin{array}{c}\text { Field } \\
{\left[{ }^{\prime}\right]} \\
(3)\end{array}$ & $\begin{array}{c}\text { Scale } \\
{\left[{ }^{\prime \prime} / \text { pix }\right]} \\
(4)\end{array}$ & Detector & $\begin{array}{c}\text { Seeing } \\
\quad\left[{ }^{\prime \prime}\right] \\
(6)\end{array}$ \\
\hline \multicolumn{6}{|c|}{ Optical } \\
\hline Mar. 1996 & 1.1 LO & 4.9 & $0.729^{a)}$ & TI 800 & 2.0 \\
\hline May 1996 & $1.0 \mathrm{HL}$ & 27.0 & 0.825 & LORAL 2048 & 3.0 \\
\hline Jun. 1996 & $1.2 \mathrm{CA}$ & 8.6 & 0.503 & TEK 1024 & 2.0 \\
\hline Aug. 1996 & $1.0 \mathrm{HL}$ & 27.0 & 0.825 & LORAL 2048 & 3.0 \\
\hline Sep. 1996 & $1.2 \mathrm{CA}$ & 8.6 & 0.503 & TEK 1024 & 2.0 \\
\hline Dec. 1996 & $0.6 \mathrm{BO}$ & 4.8 & 0.496 & TH 7882 & 1.8 \\
\hline Jan. 1997 & $1.2 \mathrm{CA}$ & 8.6 & 0.503 & TEK 1024 & 2.0 \\
\hline Feb. 1997 & $1.0 \mathrm{HL}$ & 6.8 & $0.800^{\mathrm{a})}$ & LORAL 2048 & 3.2 \\
\hline Apr. 1997 & $1.5 \mathrm{DA}$ & 13.7 & 0.390 & LORAL 2048 & 1.5 \\
\hline May 1997 & $1.1 \mathrm{LO}$ & 9.9 & $0.730^{\mathrm{a})}$ & SITe $2 \mathrm{k}$ & 2.0 \\
\hline Sep. 1997 & $1.0 \mathrm{HL}$ & 27.0 & 0.825 & LORAL 2048 & 3.0 \\
\hline Jun. 1998 & $1.5 \mathrm{DA}$ & 13.7 & 0.390 & LORAL 2048 & 1.6 \\
\hline \multicolumn{6}{|c|}{ Near Infrared } \\
\hline Mar. 1996 & $1.2 \mathrm{CA}$ & 5.1 & 1.200 & NICMOS3 & 1.8 \\
\hline Sep. 1996 & $2.2 \mathrm{CA}$ & 2.7 & 0.642 & NICMOS3 & 1.8 \\
\hline Jan. 1997 & $2.2 \mathrm{CA}$ & 2.7 & 0.642 & NICMOS3 & 1.5 \\
\hline Apr. 1997 & $2.2 \mathrm{ESO}$ & 3.0 & 0.708 & NICMOS3 & 1.6 \\
\hline Feb. 1998 & $2.2 \mathrm{CA}$ & 2.7 & 0.642 & NICMOS3 & 1.8 \\
\hline May 1998 & $2.2 \mathrm{ESO}$ & 3.0 & 0.708 & NICMOS3 & 1.5 \\
\hline
\end{tabular}

a) Pixel binning $2 \times 2$.

we could make use of observations obtained under nonphotometric conditions. For that reason the optical and near infrared contour maps of the sample galaxies shown in Figs. 4 and 5 are not flux calibrated.

As for the optical observations, the telescope/detector characteristics as well as a list of all galaxies observed in the near infrared are given in Tables 2 and 4, respectively.

\subsection{Data reduction}

The optical data were reduced using the MIDAS software package, developed by ESO. Following the standard reduction procedures (bias subtraction, flat fielding with sky flats) the remaining gradients in the background of galaxies that covered a major fraction of the field of view were removed using a two-dimensional polynomial. For some of the frames that were affected by bad columns these columns were also removed by the standard MIDAS fitting routine. In order to increase the signal-to-noise ratio
$\mathrm{S} / \mathrm{N}$ (important for an investigation of faint disk features) the images of fainter objects were binned $(2 \times 2)$.

Finally, the frames were rotated in such a way that the galaxy planes are in a horizontal position (assuming symmetrical light distribution of the vertical disk profiles). It should be stressed that the remaining small rotation error - which is typically $\pm 0.4^{\circ}$ for most of the relatively uniform disks of non-interacting galaxies - can be considered by the disk fitting routine (Sect. 4). For galactic disks affected by strong disk warping (mostly interacting objects) precise rotation was more difficult. For these cases, only the inner parts of the galaxy disk were considered to determine the rotation.

For standard reduction of the near infrared data, the IRAF software package was used. In particular, the sky frame subtraction, the flat fielding, and the combining of the flat-fielded images was done with the ARNICA (Arcetri Near-Infrared Camera) add-on package. The sky frames used for the flat field subtraction were obtained from a set of the nearest frames in time, filtered by a median. The median filtering also removed the stars in the sky frames. To produce a final source frame, the reduced, flat-fielded images were combined using the ARNICA standard "mosaic" task (this task includes both median filtering, and centering of frames by stars in the field).

Due to the small detector size - the resulting field of view was $\approx 3^{\prime}$ on average (Table 2 ) - images of larger fields were produced by mosaicing. Since this is a time-expensive procedure, it was only applied in order to obtain images of some larger objects. For precise adjustment of each of the frames we used either stars in the field or the sharp central bulge regions of the galaxies themselves.

Image rotation was applied as explained for the optical images.

Isophote maps of the complete samples of interacting/merging and non-interacting galaxies, respectively, are shown in Figs. 4 and 5 of this paper.

\section{Disk modelling}

\subsection{Overview on the disk model properties}

In order to analyze and to compare the radial and vertical disk structure of a large sample of highly-inclined/edgeon spiral galaxies, it is necessary to apply a disk model that enables both a good quantitative and very flexible description of the 3-dimensional luminosity distribution. Although mathematical simplicity is also a desired property of disk models, there should be a firm physical basis.

Therefore, a disk modelling- and fitting procedure was developed based on the results of a fundamental study of edge-on spiral galaxies by van der Kruit \& Searle (1981a,b; $1982 \mathrm{a}, \mathrm{b})$ as well as on other observational studies thereafter (e.g. van der Kruit 1988; Barteldrees \& Dettmar 
1994; de Grijs \& van der Kruit 1996; Just et al. 1996). The disk model presented here also considers the effects of an inclined disk $\left(i \neq 90^{\circ}\right)$ as well as 3 different vertical luminosity distributions.

Using the following notation for disk model parameters

$\begin{array}{ll}L_{0} & \ldots \text { central luminosity density } \\ i & \ldots \text { inclination angle of the disk } \\ R_{\max } & \ldots \text { disk cut-off Radius } \\ h & \ldots \text { disk scale length } \\ z_{0} & \ldots \text { disk scale height } \\ f_{n}\left(z, z_{0}\right) & \ldots \text { vertical distribution, see }(2) \text { to }(4) \\ \Theta\left(R_{\max }-r\right) & \ldots \text { truncation (Heaviside-) function }\end{array}$

(1 for $r<R_{\max } ; 0$ for $r \geq R_{\max }$ )

the 3-dimensional luminosity distribution $L_{n}(n=1,2,3)$ of the disk can be described in cylindrical coordinates by

$L_{n}(r, z)=L_{0} \exp (-r / h) f_{n}\left(z, z_{0}\right) \Theta\left(R_{\max }-r\right)$.

A set of 3 functions $f_{n}\left(z, z_{0}\right)$ with the same asymptotic behaviour for $z / z_{0} \gg 1$ - proposed by van der Kruit (1988); Wainscoat et al. (1989, 1990); Burkert \& Yoshii (1996) - is used to describe the vertical luminosity distribution $L(z)$ :

$f_{1}\left(z, z_{0}\right)=4 \exp \left(-2|z| / z_{0}\right)$,

$f_{2}\left(z, z_{0}\right)=2 \operatorname{sech}\left(2 z / z_{0}\right)$,

$f_{3}\left(z, z_{0}\right)=\operatorname{sech}^{2}\left(z / z_{0}\right)$.

Thus, for large $z$, a comparison between different scale heights is possible via

$z_{0\left(\mathrm{sech}^{2}\right)}=\sqrt{2} z_{0(\mathrm{sech})}=2 z_{0(\exp )} \cdot$

The use of this combination of 3 different vertical luminosity distributions meets the mentioned specifications and allows also very flexible description of a large variety of vertical profiles of galactic disks.

In order to obtain a final disk model, i.e. a twodimensional intensity distribution, integration of (1) along the line-of-sight through the disk is required. But there exist only two possible disk models - the projection faceon $\left(i=0^{\circ}\right)$ and edge-on $\left(i=90^{\circ}\right)$, combined with the isothermal disk model (4) - which would allow an analytical solution of (1) using a modified Bessel function (van der Kruit \& Searle 1981a). Therefore, integration of (1) must be calculated numerically. Hence, the intensity $I(Y, Z)$ of one point (pixel) of the disk - as it can be seen by an observer at the projected coordinates $Y$ and $Z$ on the CCD - can be written in cartesian coordinates in the following form (for example, only the double exponential disk model (2) is given here in its explicit form, for $i \neq 0$; integration of models (3) and (4) is analogous):

$$
\begin{aligned}
I(Y, Z)= & 4 L_{0} \int_{r_{\min }}^{r_{\max }} \exp \left(-\frac{\sqrt{r^{2} \sin ^{2}(i)+Y^{2}}}{h}\right) \\
& * \exp \left(-\frac{2(|r \cos (i)+Z / \sin (i)|)}{z_{0}}\right) \mathrm{d} r .
\end{aligned}
$$

The integration limits are, in a direction of the line-ofsight, given by the geometry of the "galaxy-cylinder":

$r_{\max , \min }= \pm \sqrt{R_{\max }^{2}-Y^{2}} / \sin (i)$,

and can be approximated for $i \approx 0^{\circ}$ by

$r_{\max , \min }= \pm 10 z_{0}$,

i.e. by a cylinder with a height of about 10 scale heights. At larger distances above the disk plane the contribution of luminosity, acc. to (2)-(4), becomes negligibly. The integration of (6) along the line-of-sight through the disk"cylinder" is realized by a modified Newton-Cotes rule.

According to (1), (6), and (7) the intensity $I_{n}$ of any disk model can be described by an integral of a family of 3 different density laws $L_{n}$, depending on 5 free parameters each:

$I_{n}(Y, Z)=\int L_{n}\left(L_{0}, i, R_{\max }, h, z_{0}\right) \mathrm{d} r$.

In practice, this is still a large parameter space. It therefore requires a further, step-by-step restriction, realized in the following disk fitting procedure.

\subsection{The disk fitting procedure}

In order to derive relible disk parameters we developed two independent disk fitting procedures that were designed to comply the special tasks proposed for the first (Papers I+II) and second (Paper III) part of this study (see Sect. 1).

The first fitting procedure was realized semiautomatically. It uses different graphs enabling a direct comparison by eye of a set of radial and vertical profiles within a preselected disk area with those of an underlying disk model. To take advantage of symmetrical light distribution of galactic disks the profiles used for modelling are averaged over two quadrants. They are usually displayed equidistantly and cover the whole fitting area. The size of the fitting area vastly depends on the properties of the individual image, i.e. on both the image quality and some special features of the galaxy disk itself. Therefore the program allows - after a first inspection of the profiles - an interactive selection of a qualified fitting area. Depending on the $\mathrm{S} / \mathrm{N}$ ratio and the spatial resolution of the images it is also possible to use a desired pixel binning for both image and model (as an example Fig. 2 shows one of the sets of radial and vertical disk profiles). This kind of modelling allows fast and flexible, but reliable disk fitting of a large number of galaxies - in particular of interacting/merging galaxies - whose disk profiles do often show considerable deviations from the simple model. Such galaxies are therefore difficult to handle with conventional least squares fitting methods.

The second fitting procedure uses the same set of disk models as described before but a least square fitting in order to fit the scale height as a function of the 

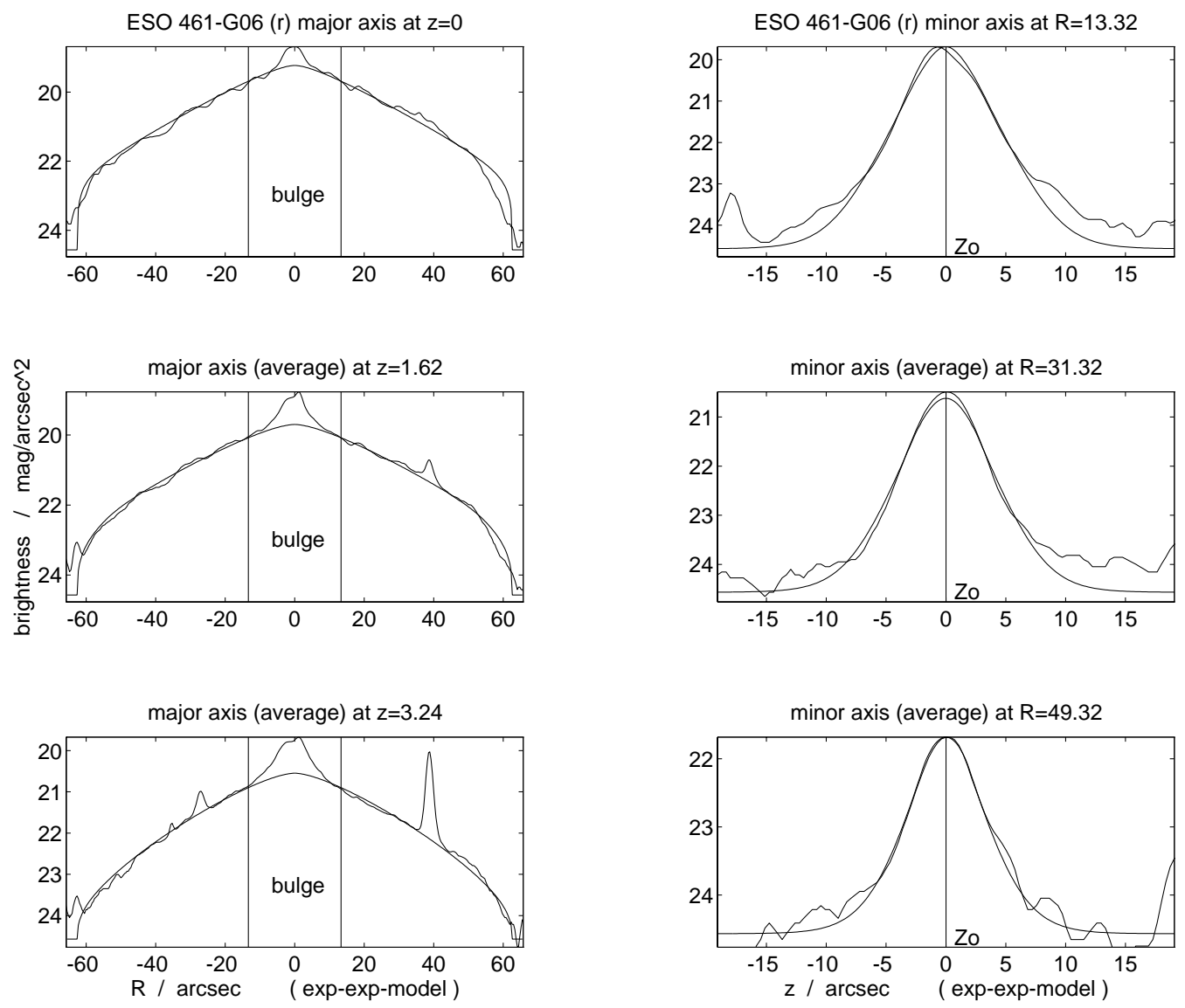

Fig. 2. Example of the disk fitting procedure: Set of typical radial (left panels) and vertical (right panels) profiles used for disk fitting of the Sc-galaxy ESO 461-G06 ( $r$-band, see also Fig. 5). The galaxy profiles (averaged) are displayed together with the final disk model, the used parameters are: $i=88.5^{\circ} ; R_{\max }=60^{\prime \prime} 8 ; h=16^{\prime \prime} .0 ; z_{0}=3^{\prime \prime} .0 ; f(z) \propto \exp$ (further explanation see text)

galactocentric distance. The properties of the program will be described in detail in Paper III. To ensure homogenity of the fitting results the fit quality reached with this method was compared with that of the above described semi-automated disk modelling (for this purpose the data of non-disturbed disk profiles of galaxies in the non-interacting sample were used). It was found that both methods give consistent results with errors on (or below) a $10 \%$-level. The errors found for the fits of the scale height only are even smaller than $5 \%$. The remaining discrepancies are not due to the individual method itself but rather to the following two reasons: first, the fitting areas are slightly different (i.e. they are usually more restricted for the least square method as a result of its sensibility against the error sources described in the following). Second, the scale height of a large fraction of galaxy disks investigated shows variations of different absolute size (both irregularly and systematically, i.e. gradients) along the galactocentric distance. Therefore this point will be discussed in detail in Paper III. A detailed comparison of the semi-automated disk modelling with another, independent developed least square fitting routine will be given in Pohlen et al. (2000).
After comparison of both fitting methods we choose to use the semi-automated disk modelling for this part of the study because it combines the advantages of fast and flexible fitting with a high accuracy. In view of the existing data the point of flexible fitting is very important since despite all structural similarities - the radial and vertical profiles of induvidual (disk) galaxies are unique. The profiles investigated here are often heavily contaminated by light from, e.g., a bulge and/or a bar, a nearby companion, other disk components, foreground stars or reflections from bright stars. In addition, the modelling may be complicated by strong dust extinction along the galactic plane, by a low $\mathrm{S} / \mathrm{N}$ ratio, or a considerably warped disk (mainly interacting/merging galaxies). Since most of the foregoing deviations can not be easily quantified their complete consideration by an automated fitting procedure thus seems almost impossible and would cause unpredictably errors.

In the following step-by-step procedure the disk parameters of the semi-automated disk fitting procedure are reduced systematically: as a first step, the inclination is determined by using the axis ratio of the dust lane in the disk plane of optical images. Given a relatively sharp 

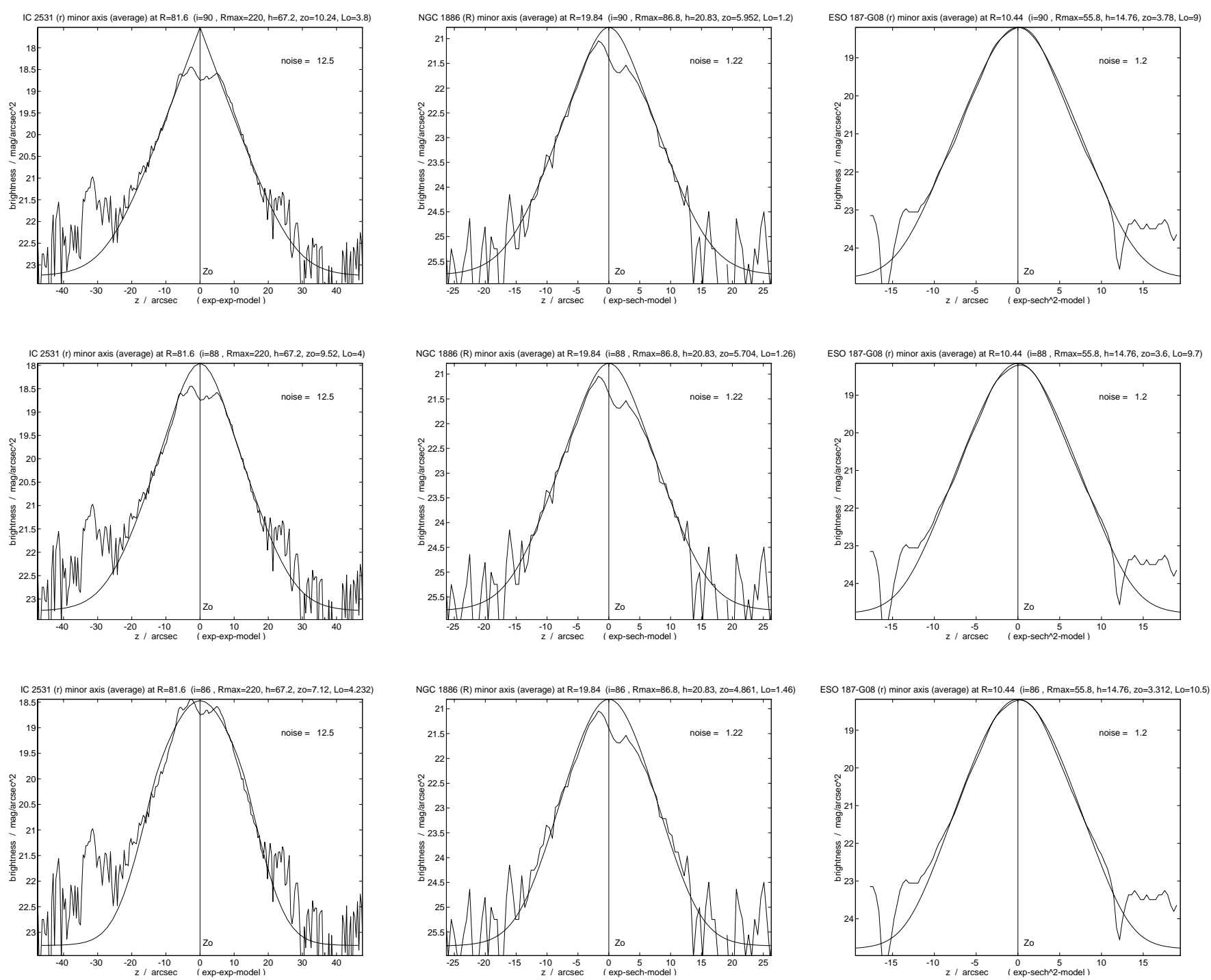

Fig. 3. The influence of inclination on the shape of vertical disk profiles, shown for 3 different edge-on galaxies and their corre-

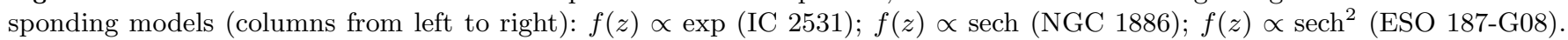
Although the disk inclination is varied between $i=86^{\circ} \ldots 90^{\circ}$, for each of the vertical profiles a quantitative good fit can be found by changing only the scale height $z_{0}$ and the central luminosity density $L_{0}$ (as indicated; further explanation see text)

dust lane, an accuracy of less than $\pm 0.5^{\circ}$ can be reached. The central luminosity density, $L_{0}$, is calculated automatically by the fitting program for each new parameter set by using a number of preselected reference points along the disk (outside the bulge- or bar-light contaminated regions).

Given a sufficient $\mathrm{S} / \mathrm{N}$ ratio, the cut-off radius $R_{\max }$ can be fitted to the major axes profiles with an accuracy between $(5-10) \%$. The cut-off is determined by a significant decrease of the intensity extrapolated to $I=0$ (left panels in Fig. 2). For highly-inclined disks such as the ones studied here, the effect of a variation of $R_{\max }$ on the slope of radial disk profiles is negligible.
Thus, the remaining "real" fitting parameters are the disk scale length and height, $h$ and $z_{0}$, as well as the set of 3 functions $f_{n}(z)$. Within the following procedure both the scale length and the scale height are fitted in an iterative process until a first good convergence of the "global" fit is achieved. During this process, the quality of the corresponding fits can be checked simultaneously using a small set of radial and vertical disk profiles (usually 3 profiles each, see Fig. 2 as an example). For further small corrections, if necessary, both parameters can be considered as independent and thus separated without any loss of accuracy. For this "fine tuning" a set with more disk profiles (usually 6-8) is used for both parameters. 
During the iterative process of modelling the scale length is fitted to a set of averaged major axes profiles in a radial region typically between $(0.7-2.8) h$ and vertically outside strong dust extinction (left panels in Fig. 2). The fit quality of the vertical profiles along the disk can be used as a cross-check for the scale length (right panels in Fig. 2). As a result of the error sources mentioned at the beginning the disk scale length of a galaxy can be reproduced (with the same method) with an accuracy of $(5-10) \%$. In contrast to this, the scale lengths derived with different methods can in some cases differ by $(25-50) \%$.

The disk scale height $z_{0}$ is estimated by fitting the $z$ profiles inside the previously selected radial regions, which are outside the bulge- or bar contamination (right panels in Fig. 2). The vertical region used for the fits is typically between $(0.2-2) z_{0}$, but depends strongly on the individual characteristic of the dust lane. If the disk inclination is known precisely, this fitting method works reliably. Otherwise, additional errors may be introduced (see next section).

The vertical disk profiles of most of the galaxies investigated in optical passbands enable a reliable choice of the quantitatively best fitting function $f_{n}(z)$. This is because deviations between different models become visible at vertical distances larger than that of the most sharplypeaked dust regions. For those disks that are affected by strong dust extinction, the choice was made easier by using a combination of both optical and near infrared profiles. For these cases profiles of both passbands were, if available, used for fitting.

\subsection{The influence of inclination on vertical disk profiles}

If a large sample of disk galaxies is investigated statistically - as is the case in this study - moderate deviations from edge-on orientation of the order of $\pm 5^{\circ}$ are common (Sect. 2). It was found in this study and in Schwarzkopf \& Dettmar (1998) that, if reliable values for the scale height are desired, deviations from $90^{\circ}$ larger than $4^{\circ}-5^{\circ}$ can not be neglegted. Therefore in this section the influence of small changes of inclination on vertical disk profiles and on the resulting parameters will be investigated in detail.

For that purpose we selected $3 r$-band images of galaxies in the non-interacting sample with well known disk parameters, but with different vertical profiles: $f(z) \propto \exp$ $(\mathrm{IC} 2531) ; f(z) \propto \operatorname{sech}(\mathrm{NGC} 1886) ;$ and $f(z) \propto \operatorname{sech}^{2}$ (ESO 187-G08). At 3 different inclinations $\left(i=90^{\circ} ; 88^{\circ}\right.$; and $86^{\circ}$ ) the vertical profiles of each model were bestfitted to the observed disk profiles (see left, middle, and right panels of Fig. 3). To compensate for the effect of inclination, both the disk scale height, $z_{0}$, and the central luminosity density, $L_{0}$, must be changed. As can be seen in the disk parameters (middle raw in Fig. 3 and Table 3 ) the effect for $z_{0}$ is, at $i=88^{\circ}$, around the $5 \%$-level for
Table 3. The influence of inclination on vertical disk profiles. Columns: (1) Disk inclination used for the model; (2), (4), (6) Error of disk scale height using $f(z) \propto \exp$, sech, and sech $^{2}$; (3), (5), (7) Error of central luminosity density using $f(z) \propto$ exp, sech, and sech ${ }^{2}$

\begin{tabular}{ccccccr}
\hline \hline Disk & \multicolumn{2}{c}{$f(z) \propto \exp$} & \multicolumn{2}{c}{$f(z) \propto$ sech } & \multicolumn{2}{c}{$f(z) \propto$ sech $^{2}$} \\
inclination & $\Delta z_{0}$ & $\Delta L_{0}$ & $\Delta z_{0}$ & $\Delta L_{0}$ & $\Delta z_{0}$ & $\Delta L_{0}$ \\
$(1)$ & $(2)$ & $(3)$ & \multicolumn{1}{c}{$(4)$} & \multicolumn{1}{c}{$(5)$} & \multicolumn{1}{c}{$(6)$} & $(7)$ \\
\hline $88^{\circ}$ & $7 \%$ & $5 \%$ & $5 \%$ & $5 \%$ & $5 \%$ & $7 \%$ \\
$86^{\circ}$ & $30 \%$ & $11 \%$ & $18 \%$ & $18 \%$ & $13 \%$ & $18 \%$ \\
\hline
\end{tabular}

all vertical models, whereas for $i=86^{\circ}$ (Fig. 3 bottom) the error amounts to $\approx 30 \%$ for the exp-model and, after all, to $\approx 13 \%-18 \%$ for the sech $^{2}$ and sech-models. At smaller inclinations the quality of all vertical fits decreases rapidly and allows no more qualitative good fits. As expected, the effect of slight changes of inclination on both the scale height and the shape of vertical profiles near the disk plane is the strongest for the exp-model (left panels in Fig. 3).

The effect shown above is due to the fact that the slope of the vertical disk profiles - in a region that is relevant for fitting (previous section) - remains nearly unchanged in the range between $i \approx 85^{\circ}-90^{\circ}$, whereas this is not true for the width of the vertical profiles. Hence, to obtain reliable disk parameters for $z_{0}$ and $L_{0}$ it is required that both
- the inclination of the disk is known precisely (within $\left.\pm 2^{\circ}\right)$ and

- a disk model with a flexible inclination is used.

Otherwise, substantial errors for $z_{0}$ and $L_{0}$ are introduced (Table 3 lists averaged errors $\Delta z_{0}$ and $\Delta L_{0}$, obtained by fitting 5 galaxies with 3 different vertical disk models each).

\section{Summary and conclusions}

Optical and near infrared photometric data of a sample of 110 highly-inclined/edge-on disk galaxies are presented. This sample consists of two subsamples of 61 non-interacting galaxies and 49 minor merging candidates. Additionally, 41 of these galaxies were observed in the near infrared.

The sample selection, observations, and data reduction are described. We show that - although the subsamples are naturally slightly polluted due to unavoidable selection effects - the distribution of their morphological types is almost indistinguishable, covering the range between $0 \leq T \leq 9$. This is important for the forthcoming detailed statistical study focused on the influence of interaction and minor merger on the radial and vertical disk structure of spiral galaxies. 
Moreover, a 3-dimensional disk modelling- and fitting procedure is described in order to analyze and to compare the disk structure of our sample galaxies by using characteristical parameters. We find that the vertical brightness profiles of modelled galaxy disks respond very sensitive even to small changes of inclination around perfect edge-on orientation. Therefore, projection effects of highly-inclined disks must be considered.

Acknowledgements. This work was supported by Deutsche Forschungsgemeinschaft, DFG, under grant No. GRK 118/2.

This research has made use of the NASA/IPAC Extragalactic Database (NED).

\section{References}

Arp H.C., 1966, Atlas of Peculiar Galaxies, California Institute of Technology, Pasadena

Arp H.C., Madore B.F., 1987, A Catalogue of Southern Peculiar Galaxies and Associations, Vols. I \& II. Cambridge University Press

Barteldrees A., Dettmar R.-J., 1994, A\&AS 103, 475

Burkert A., Yoshii Y., 1996, MNRAS 282, 1349

Corwin H.G., de Vaucouleurs A., de Vaucouleurs G., 1985, Southern Galaxy Catalogue (SGC). University Texas Press, Austin

Darling D.A., 1957, Ann. Math. Statist. 28, 823

Goad J.W., Roberts M.S., 1981, ApJ 250, 79

Grijs R. de, Kruit P.C. van der, 1996, A\&AS 117, 19

Grijs R. de, 1997, Ph.D. Thesis, University Groningen

Griv E., Peter W., 1996a, ApJ 469, 84

Griv E., Peter W., 1996b, ApJ 469, 99

Griv E., Peter W., 1996c, ApJ 469, 103

Hubble E.P., 1926, ApJ 64, 321

Just A., Fuchs B., Wielen R., 1996, A\&A 309, 715

Kruit P.C. van der, Searle L., 1981a, A\&A 95, 105

Kruit P.C. van der, Searle L., 1981b, A\&A 95, 116

Kruit P.C. van der, Searle L., 1982a, A\&A 110, 61

Kruit P.C. van der, Searle L., 1982b, A\&A 110, 79

Kruit P.C. van der, 1988, A\&A 192, 117
Lauberts A., Valentijn E.A., 1989, The Surface Photometry Catalogue of the ESO-Uppsala Galaxies, European Southern Observatory

Mihos J.C., Walker I.R., Hernquist L., 1995, ApJ 447, L87

Nilson P., 1973, Uppsala General Catalogue of Galaxies, Uppsala University

Pohlen M., Dettmar R.-J., Lütticke R., Schwarzkopf U., 2000, A\&AS (submitted)

Quinn P.J., Hernquist L., Fullagar D.P., 1993, ApJ 403, 74

Reshetnikov V., Combes F., 1996, A\&AS 116, 417

Reshetnikov V., Combes F., 1997, A\&A 324, 80

Sachs L., 1992, Angewandte Statistik. Springer-Verlag Berlin Heidelberg

Sandage A., 1961, The Hubble Atlas of Galaxies. Institution of Washington Publication, p. 618

Sandage A., Bedke J., 1988, Atlas of Galaxies useful for measuring the cosmological distance scale, Washington

Sandage A., Bedke J., 1994, The Carnegie Atlas of Galaxies, Vols. I, II, Carnegie Institution of Washington Publication, p. 638

Schwarzkopf U., Dettmar R.-J., 1998, in: The Magellanic Clouds and Other Dwarf Galaxies, Braun J.M., Richtler T. (eds.). Shaker Publ., Aachen, p. 297

Schwarzkopf U., Dettmar R.-J., 2000, A\&A (submitted) (Paper II)

Toth G., Ostriker J.P., 1992, ApJ 389, 5

Vaucouleurs G. de, Vaucouleurs A. de, Corwin Jr. H.G., Paturel G., Fouque P., 1991, Third Reference Catalogue of Bright Galaxies, Vols. I-III. Springer

Velazquez H., White S.D.M., 1999, MNRAS 304, 254

Wainscoat R.J., Freeman K.C., Hyland A.R., 1989, ApJ 337, 163

Wainscoat R.J., Hyland A.R., Freeman K.C., 1990, ApJ 348, 85

Walker I.R., Mihos C., Hernquist L., 1996, ApJ 460, 121

Zaritsky D., Smith R., Frenk C., White S.D.M., 1993, ApJ 405, 464

Zaritsky D., 1995, ApJ 448, L17

Zaritsky D., 1996, ApJ 462, 50

Zaritsky D., Smith R., Frenk C., White S.D.M., 1997, ApJ 478, 39 
Table 4. Complete list of the optical/near infrared galaxy samples used in this study. Columns: (1) Serial number; (2) Galaxy name; (3), (4) Center coordinates for epoch 2000, based on NASA/IPAC Extragalactic Database (NED); (5) Telescopes used: $2.2 \mathrm{CA}=2.2 \mathrm{~m}$ Calar Alto, $2.2 \mathrm{ESO}=2.2 \mathrm{~m} \mathrm{ESO} / \mathrm{MPIA}, 1.5 \mathrm{DA}=1.54 \mathrm{~m}$ Danish, $1.2 \mathrm{CA}=1.23 \mathrm{~m}$ Calar Alto, $1.1 \mathrm{LO}=$ $1.1 \mathrm{~m}$ Lowell, 1.0 HL = $1.06 \mathrm{~m}$ Hoher List, 0.6 BO = $0.6 \mathrm{~m}$ Bochum; (6) Observing dates (mm dd yy); (7) Passband used; (8) Exposure time (total); (9) Total blue magnitude (if available); (10) Revised Hubble type, based on NED, according to Table 1; (11) Notes: 1 = data of this paper, 2 = supplementary data from Barteldrees \& Dettmar (1994)

\begin{tabular}{ccccccccccc}
\hline \hline No. & Galaxy & RA $(2000)$ & Dec $(2000)$ & Telescope & Date & Band & Exp.-time & $B_{\mathrm{T}}$ & Type & Note \\
& & $\mathrm{h}$ & $\mathrm{m}$ & $\mathrm{s}$ & $\circ$ & $\prime \prime$ & & & & \\
{$[\mathrm{min}]$} & & & \\
$(1)$ & $(2)$ & $(3)$ & $(4)$ & $(5)$ & $(6)$ & $(7)$ & $(8)$ & $(9)$ & $(10)$ & $(11)$ \\
\hline
\end{tabular}

Interacting / Merging

\begin{tabular}{|c|c|c|c|c|c|c|c|c|c|c|}
\hline 1 & NGC 7 & 000820.6 & -295454 & $0.6 \mathrm{BO}$ & Dec. 2796 & $R$ & 75 & 14.40 & 4.5 & 1 \\
\hline 2 & UGC 260 & 002703.0 & +113503 & $1.0 \mathrm{HL}$ & Sep. 0497 & $R$ & 65 & 13.71 & 6.0 & 1 \\
\hline \multirow[t]{2}{*}{3} & NGC 128 & 002915.0 & +025155 & $1.2 \mathrm{CA}$ & Sep. 0896 & $R$ & 30 & 12.77 & -2.0 & 1 \\
\hline & & & & $2.2 \mathrm{CA}$ & Sep. 0396 & $K$ & 4 & - & -2.0 & 1 \\
\hline 4 & AM 0107-375 & 010942.0 & -374227 & $0.6 \mathrm{BO}$ & Dec. 2896 & $R$ & 65 & - & 3.5 & 1 \\
\hline 5 & ESO 296-G17 & 012355.0 & -380044 & $0.6 \mathrm{BO}$ & Dec. 2996 & $R$ & 60 & 16.38 & 3.0 & 1 \\
\hline 6 & ESO 354-G05 & 015207.0 & -333146 & $0.6 \mathrm{BO}$ & Jan. 0597 & $R$ & 75 & 15.95 & 4.0 & 1 \\
\hline 7 & ESO 245-G10 & 015644.0 & -435823 & $0.6 \mathrm{BO}$ & Dec. 3196 & $R$ & 75 & 14.28 & 3.0 & 1 \\
\hline 8 & ESO 417-G08 & 025847.0 & -320552 & $0.6 \mathrm{BO}$ & Jan. 0497 & $R$ & 75 & 13.64 & 0.7 & 1 \\
\hline 9 & ESO 199-G12 & 030325.0 & -502943 & $0.6 \mathrm{BO}$ & Dec. 2896 & $R$ & 88 & 15.52 & 8.0 & 1 \\
\hline 10 & ESO $357-G 16$ & 031934.0 & -322753 & $0.6 \mathrm{BO}$ & Jan. 0297 & $R$ & 75 & 14.34 & 3.0 & 1 \\
\hline 11 & ESO 357-G26 & 032356.0 & -362750 & $1.2 \mathrm{CA}$ & Jan. 0197 & $R$ & 75 & 11.37 & -1.0 & 1 \\
\hline 12 & ESO 418-G15 & 033923.0 & -311919 & $0.6 \mathrm{BO}$ & Dec. 2896 & $R$ & 55 & 12.40 & 4.0 & 1 \\
\hline 13 & NGC $1531 / 32$ & 041159.0 & -325057 & $1.5 \mathrm{DA}$ & Apr. 0897 & $r$ & 30 & 10.65 & 2.7 & 1 \\
\hline 14 & ESO 202-G04 & 041746.0 & -500951 & $0.6 \mathrm{BO}$ & Dec. 2696 & $R$ & 80 & 13.47 & 2.0 & 1 \\
\hline 15 & ESO $362-G 11$ & 051639.0 & -370600 & $0.6 \mathrm{BO}$ & Jan. 0597 & $R$ & 75 & 13.04 & 4.0 & 1 \\
\hline \multirow[t]{2}{*}{16} & NGC 1888 & 052235.0 & -112958 & $1.5 \mathrm{DA}$ & Apr. 0897 & $r$ & 40 & 12.83 & 5.0 & 1 \\
\hline & & & & $2.2 \mathrm{ESO}$ & Apr. 1097 & $K^{\prime}$ & 40 & - & 5.0 & 1 \\
\hline 17 & ESO 363-G07 & 053313.0 & -362359 & $0.6 \mathrm{BO}$ & Dec. 3096 & $R$ & 75 & 13.25 & 5.5 & 1 \\
\hline 18 & ESO 487-G35 & 054201.0 & -225643 & $0.6 \mathrm{BO}$ & Jan. 0197 & $R$ & 90 & 13.39 & 7.8 & 1 \\
\hline \multirow[t]{2}{*}{19} & NGC 2188 & 061010.0 & -340622 & $1.5 \mathrm{DA}$ & Apr. 0997 & $r$ & 75 & 12.14 & 9.0 & 1 \\
\hline & & & & $2.2 \mathrm{ESO}$ & Apr. 1297 & $K^{\prime}$ & 33 & - & 9.0 & 1 \\
\hline \multirow[t]{2}{*}{20} & UGC 3697 & 071121.3 & +715006 & $1.1 \mathrm{LO}$ & Mar. 1696 & $R$ & 33 & 13.50 & 7.0 & 1 \\
\hline & & & & $1.2 \mathrm{CA}$ & Mar. 0196 & $H$ & 53 & - & 7.0 & 1 \\
\hline 21 & ESO 060-G24 & 090240.3 & -681338 & $1.5 \mathrm{DA}$ & Jun. 0398 & $r$ & 30 & 13.95 & 2.5 & 1 \\
\hline 22 & ESO 497-G14 & 090742.0 & -233715 & $0.6 \mathrm{BO}$ & Dec. 2696 & $R$ & 75 & 14.16 & 3.0 & 1 \\
\hline 23 & NGC 2820 & 092147.0 & +641529 & $1.2 \mathrm{CA}$ & Mar. 0196 & $H$ & 53 & 13.28 & 5.0 & 1 \\
\hline \multirow[t]{2}{*}{24} & NGC 3044 & 095340.0 & +013446 & $1.5 \mathrm{DA}$ & Apr. 0897 & $r$ & 45 & 12.46 & 5.0 & 1 \\
\hline & & & & $2.2 \mathrm{ESO}$ & Apr. 1097 & $K^{\prime}$ & 40 & - & 5.0 & 1 \\
\hline \multirow[t]{2}{*}{25} & NGC 3187 & 101748.0 & +215225 & $1.5 \mathrm{DA}$ & Apr. 0997 & $r$ & 30 & 13.91 & 5.0 & 1 \\
\hline & & & & $2.2 \mathrm{ESO}$ & Apr. 1197 & $K^{\prime}$ & 28 & - & 5.0 & 1 \\
\hline 26 & ESO $317-G 29$ & 102744.0 & -402608 & $0.6 \mathrm{BO}$ & Dec. 2896 & $R$ & 50 & 13.74 & 1.0 & 1 \\
\hline \multirow[t]{2}{*}{27} & ESO 264-G29 & 104012.0 & -470611 & $0.6 \mathrm{BO}$ & Jan. 0696 & $R$ & 60 & 15.68 & 5.6 & 1 \\
\hline & & & & $2.2 \mathrm{ESO}$ & Apr. 1297 & $K^{\prime}$ & 11 & - & 5.6 & 1 \\
\hline \multirow[t]{2}{*}{28} & NGC 3432 & 105231.0 & +363708 & $1.1 \mathrm{LO}$ & May 3197 & $R$ & 40 & 11.67 & 9.0 & 1 \\
\hline & & & & $2.2 \mathrm{CA}$ & Feb. 1398 & $K^{\prime}$ & 60 & - & 9.0 & 1 \\
\hline \multirow[t]{2}{*}{29} & NGC 3628 & 112016.0 & +133522 & $1.5 \mathrm{DA}$ & Apr. 0997 & $r$ & 95 & 10.28 & 3.0 & 1 \\
\hline & & & & $2.2 \mathrm{CA}$ & Feb. 1598 & $K^{\prime}$ & 13 & - & 3.0 & 1 \\
\hline \multirow[t]{2}{*}{30} & ESO 378-G13 & 113708.0 & -324913 & $0.6 \mathrm{BO}$ & Jan. 0797 & $R$ & 60 & 15.45 & 1.0 & 1 \\
\hline & & & & $2.2 \mathrm{ESO}$ & Apr. 1297 & $K^{\prime}$ & 38 & - & 1.0 & 1 \\
\hline \multirow[t]{2}{*}{31} & ESO 379-G20 & 120059.0 & -351136 & $0.6 \mathrm{BO}$ & Jan. 0897 & $R$ & 75 & 15.44 & 1.0 & 1 \\
\hline & & & & $2.2 \mathrm{ESO}$ & Apr. 1197 & $K^{\prime}$ & 36 & - & 1.0 & 1 \\
\hline \multirow[t]{2}{*}{32} & NGC 4183 & 121318.0 & +434155 & $1.1 \mathrm{LO}$ & Jun. 0297 & $R$ & 25 & 12.86 & 6.0 & 1 \\
\hline & & & & $2.2 \mathrm{CA}$ & Feb. 1498 & $K^{\prime}$ & 40 & - & 6.0 & 1 \\
\hline \multirow[t]{2}{*}{33} & NGC 4631 & 124208.0 & +323228 & $1.1 \mathrm{LO}$ & May 3197 & $R$ & 30 & 9.75 & 7.0 & 1 \\
\hline & & & & $2.2 \mathrm{CA}$ & Feb. 1698 & $K^{\prime}$ & 13 & - & 7.0 & 1 \\
\hline
\end{tabular}


Table 4. continued

\begin{tabular}{|c|c|c|c|c|c|c|c|c|c|c|}
\hline No. & $\begin{array}{c}\text { Galaxy } \\
\text { (2) }\end{array}$ & $\begin{array}{cl}\mathrm{RA} & (2000) \\
\mathrm{h} & \mathrm{m} \quad \mathrm{s} \\
& (3)\end{array}$ & $\begin{array}{c}\operatorname{Dec}(2000) \\
\circ \quad, \quad \\
(4)\end{array}$ & (5) & (6) & Band & $\begin{array}{c}\text { Exp.-time } \\
\text { [min] } \\
(8)\end{array}$ & $\begin{array}{c}B_{\mathrm{T}} \\
{[\mathrm{mag}]} \\
(9)\end{array}$ & Type & Note \\
\hline 34 & NGC 4634 & 124240.4 & +141747 & $\begin{array}{l}1.5 \mathrm{DA} \\
2.2 \mathrm{CA}\end{array}$ & $\begin{array}{l}\text { Jun. } 0398 \\
\text { Feb. } 1598\end{array}$ & $\begin{array}{c}r \\
K^{\prime}\end{array}$ & $\begin{array}{l}45 \\
25\end{array}$ & $\begin{array}{r}13.16 \\
-\end{array}$ & $\begin{array}{l}6.0 \\
6.0\end{array}$ & $\begin{array}{l}1 \\
1\end{array}$ \\
\hline 35 & NGC 4747 & 125145.0 & +254627 & $1.1 \mathrm{LO}$ & Jun. 0297 & $R$ & 72 & 12.96 & 6.0 & 1 \\
\hline 36 & NGC 4762 & 125256.3 & +111348 & $\begin{array}{l}1.5 \mathrm{DA} \\
2.2 \mathrm{CA}\end{array}$ & $\begin{array}{l}\text { Apr. } 0897 \\
\text { Feb. } 1598\end{array}$ & $\begin{array}{c}r \\
K^{\prime}\end{array}$ & $\begin{array}{l}30 \\
27\end{array}$ & 11.12 & $\begin{array}{l}-2.0 \\
-2.0\end{array}$ & $\begin{array}{l}1 \\
1\end{array}$ \\
\hline 37 & ESO 443-G21 & 125946.0 & -293558 & $\begin{array}{l}1.5 \mathrm{DA} \\
2.2 \mathrm{ESO}\end{array}$ & $\begin{array}{l}\text { Apr. } 0897 \\
\text { Apr. } 1197\end{array}$ & $\stackrel{r}{K^{\prime}}$ & $\begin{array}{l}45 \\
15\end{array}$ & $\stackrel{14.41}{-}$ & $\begin{array}{l}6.0 \\
6.0\end{array}$ & $\begin{array}{l}1 \\
1\end{array}$ \\
\hline 38 & NGC 5126 & 132454.0 & -302000 & $0.6 \mathrm{BO}$ & Jan. 1097 & $R$ & 40 & 14.06 & 0.0 & 1 \\
\hline 39 & ESO 324-23 & 132729.0 & -381026 & $\begin{array}{l}1.5 \mathrm{DA} \\
2.2 \mathrm{ESO}\end{array}$ & $\begin{array}{l}\text { Apr. } 0897 \\
\text { Apr. } 1097\end{array}$ & $\begin{array}{c}r \\
K^{\prime}\end{array}$ & $\begin{array}{l}45 \\
32\end{array}$ & $\begin{array}{r}13.07 \\
-\end{array}$ & $\begin{array}{l}6.5 \\
6.5\end{array}$ & $\begin{array}{l}1 \\
1\end{array}$ \\
\hline 40 & ESO 383-G05 & 132923.8 & -341623 & $1.5 \mathrm{DA}$ & Jun. 0398 & $r$ & 45 & 14.21 & 3.7 & 1 \\
\hline 41 & NGC 5297 & 134624.0 & +435225 & $1.1 \mathrm{LO}$ & Jun. 0397 & $R$ & 3 & 12.47 & 4.5 & 1 \\
\hline 42 & ESO 445-G63 & 135207 & -304941 & $1.5 \mathrm{DA}$ & Jun. 0398 & $r$ & 40 & 15.78 & 5.3 & 1 \\
\hline 43 & NGC 5529 & 141534.1 & +361336 & $\begin{array}{l}1.1 \mathrm{LO} \\
1.2 \mathrm{CA}\end{array}$ & $\begin{array}{l}\text { Jun. } 0197 \\
\text { Mar. } 0596\end{array}$ & $\begin{array}{l}R \\
H\end{array}$ & $\begin{array}{l}50 \\
12\end{array}$ & 12.75 & $\begin{array}{l}5.0 \\
5.0\end{array}$ & $\begin{array}{l}1 \\
1\end{array}$ \\
\hline 44 & NGC 5965 & 153402.0 & +564110 & $1.1 \mathrm{LO}$ & Jun. 0397 & $R$ & 60 & 12.60 & 3.0 & 1 \\
\hline 45 & NGC 6045 & 160508.0 & +174522 & $\begin{array}{l}1.2 \mathrm{CA} \\
2.2 \mathrm{CA}\end{array}$ & $\begin{array}{l}\text { Sep. } 0696 \\
\text { Sep. } 0396\end{array}$ & $\begin{array}{l}R \\
K\end{array}$ & $\begin{array}{l}30 \\
30\end{array}$ & $\begin{array}{r}14.87 \\
-\end{array}$ & $\begin{array}{l}5.0 \\
5.0\end{array}$ & $\begin{array}{l}1 \\
1\end{array}$ \\
\hline 46 & NGC 6361 & 171840.0 & +603632 & $\begin{array}{l}1.2 \mathrm{CA} \\
1.0 \mathrm{HL} \\
2.2 \mathrm{CA}\end{array}$ & $\begin{array}{l}\text { Jun. } 0296 \\
\text { May } 2096 \\
\text { Sep. } 0396\end{array}$ & $\begin{array}{c}R \\
n f^{\mathrm{a})} \\
K\end{array}$ & $\begin{array}{c}37 \\
5 \\
20\end{array}$ & $\begin{array}{r}13.87 \\
- \\
-\end{array}$ & $\begin{array}{l}3.0 \\
3.0 \\
3.0\end{array}$ & $\begin{array}{l}1 \\
1 \\
1\end{array}$ \\
\hline 47 & Arp 121 & 005924.0 & -044813 & $\begin{array}{l}1.2 \mathrm{CA} \\
2.2 \mathrm{CA}\end{array}$ & $\begin{array}{l}\text { Sep. } 0496 \\
\text { Sep. } 0496\end{array}$ & $\begin{array}{l}R \\
K\end{array}$ & $\begin{array}{l}60 \\
16\end{array}$ & - & $\begin{array}{l}2.0 \\
2.0\end{array}$ & $\begin{array}{l}1 \\
1\end{array}$ \\
\hline $\begin{array}{l}48 \\
49\end{array}$ & $\begin{array}{l}\text { ESO 462-G07 } \\
\text { IC } 4991\end{array}$ & $\begin{array}{lll}20 & 18 & 23 \\
20 & 18 & 23.0\end{array}$ & $\begin{array}{lll}-27 & 27 & 18 \\
-41 & 03 & 01\end{array}$ & $\begin{array}{l}2.2 \mathrm{ESO} \\
1.5 \mathrm{DA}\end{array}$ & $\begin{array}{l}\text { Apr. } 1297 \\
\text { Apr. } 0898\end{array}$ & $\begin{array}{c}K^{\prime} \\
r\end{array}$ & $\begin{array}{l}41 \\
30\end{array}$ & $\begin{array}{l}15.48 \\
11.56\end{array}$ & $\begin{array}{r}4.0 \\
-2.0\end{array}$ & $\begin{array}{l}1 \\
1\end{array}$ \\
\hline
\end{tabular}

Non - Interacting

\begin{tabular}{|c|c|c|c|c|c|c|c|c|c|c|}
\hline \multirow[t]{2}{*}{1} & UGC 231 & 002402.6 & +162909 & $1.0 \mathrm{HL}$ & Aug. 1996 & $R$ & 30 & 13.91 & 6.0 & 1 \\
\hline & & & & $2.2 \mathrm{CA}$ & Sep. 0596 & $K$ & 40 & - & 6.0 & 1 \\
\hline 2 & ESO $150-G 07$ & 002537.0 & -571128 & $1.5 \mathrm{DA}$ & Jun. 0498 & $r$ & 30 & 15.28 & 1.0 & 1 \\
\hline 3 & ESO 112-G04 & 002804.0 & -580613 & $2.2 \mathrm{ESO}$ & June 87 & $r$ & & 15.86 & 5.6 & 2 \\
\hline 4 & ESO $150-G 14$ & 003638.0 & -565424 & $2.2 \mathrm{ESO}$ & June 87 & $r$ & & 14.90 & 0.4 & 2 \\
\hline 5 & UGC 711 & 010837.0 & +013829 & $0.6 \mathrm{Bo}$ & Dec. 2596 & $R$ & 118 & 14.39 & 6.7 & 1 \\
\hline 6 & ESO 244-G48 & 013909.0 & -470742 & $1.5 \mathrm{DA}$ & Jun. 0398 & $r$ & 30 & 15.55 & -2.0 & 1 \\
\hline 7 & UGC 1839 & 022230.2 & -003707 & $1.2 \mathrm{CA}$ & Sep. 0896 & $R$ & 75 & 15.26 & 7.3 & 1 \\
\hline \multirow[t]{2}{*}{8} & NGC 891 & 022233.1 & +422048 & $1.0 \mathrm{HL}$ & Sep. 0597 & $R$ & 30 & 10.81 & 3.0 & 1 \\
\hline & & & & $2.2 \mathrm{CA}$ & Feb. 1498 & $K^{\prime}$ & 20 & - & 3.0 & 1 \\
\hline 9 & ESO 416-G25 & 024841.0 & -313210 & $2.2 \mathrm{ESO}$ & June 87 & $r$ & & 14.64 & 3.0 & 2 \\
\hline 10 & UGC 2411 & 025800.9 & +754500 & $1.1 \mathrm{LO}$ & Mar. 1796 & $R$ & 20 & 16.50 & 8.5 & 1 \\
\hline 11 & IC 1877 & 030310.0 & -503043 & $0.6 \mathrm{BO}$ & Dec. 2896 & $R$ & 88 & 16.30 & 3.0 & 1 \\
\hline 12 & ESO 201-G22 & 040900.4 & -484335 & $0.6 \mathrm{BO}$ & Dec. 2596 & $R$ & 148 & 14.69 & 5.0 & 1 \\
\hline \multirow[t]{2}{*}{13} & NGC 1886 & 051248.7 & -234845 & $0.6 \mathrm{BO}$ & Jan. 0697 & $R$ & 30 & 13.60 & 3.5 & 1 \\
\hline & & & & $2.2 \mathrm{CA}$ & Feb. 1598 & $K^{\prime}$ & 20 & - & 3.5 & 1 \\
\hline \multirow[t]{2}{*}{14} & UGC 3474 & 063200.6 & +713300 & $1.1 \mathrm{LO}$ & Mar. 1796 & $R$ & 10 & 15.40 & 6.0 & 1 \\
\hline & & & & $2.2 \mathrm{CA}$ & Feb. 1498 & $K^{\prime}$ & 27 & - & 6.0 & 1 \\
\hline 15 & NGC 2310 & 065353.6 & -405144 & $0.6 \mathrm{BO}$ & Jan. 0507 & $R$ & 30 & 12.74 & -2.0 & 1 \\
\hline \multirow[t]{2}{*}{16} & UGC 4278 & 081359.0 & +455443 & $1.1 \mathrm{LO}$ & Mar. 1696 & $R$ & 10 & 13.07 & 7.0 & 1 \\
\hline & & & & $1.2 \mathrm{CA}$ & Mar. 0296 & $H$ & 67 & - & 7.0 & 1 \\
\hline 17 & ESO 564-G27 & 091154.4 & -200703 & $2.2 \mathrm{ESO}$ & June 87 & $r$ & & 14.35 & 6.3 & 2 \\
\hline \multirow[t]{2}{*}{18} & UGC 4943 & 091958.1 & +371127 & $1.1 \mathrm{LO}$ & Mar. 1696 & $R$ & 6 & 14.80 & 3.0 & 1 \\
\hline & & & & $1.2 \mathrm{CA}$ & Mar. 0696 & $H$ & 8 & - & 3.0 & 1 \\
\hline 19 & IC 2469 & 092300.9 & -322659 & $1.5 \mathrm{DA}$ & Jun. 0208 & $r$ & 5 & 13.03 & 2.0 & 1 \\
\hline \multirow[t]{2}{*}{20} & UGC 5341 & 095636.6 & +203853 & $0.6 \mathrm{BO}$ & Jan. 0297 & $R$ & 134 & 15.03 & 6.0 & 1 \\
\hline & & & & $2.2 \mathrm{CA}$ & Feb. 1598 & $K^{\prime}$ & 27 & - & 6.0 & 1 \\
\hline
\end{tabular}


Table 4. continued

\begin{tabular}{|c|c|c|c|c|c|c|c|c|c|c|}
\hline No. & $\begin{array}{c}\text { Galaxy } \\
\text { (2) }\end{array}$ & 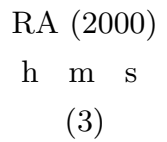 & $\begin{array}{c}\text { Dec. }(2000) \\
\circ \quad, \quad \\
(4)\end{array}$ & Telescope & Date & Band & $\begin{array}{l}\text { Exp.-time } \\
\text { [min] } \\
(8)\end{array}$ & $\begin{array}{c}B_{\mathrm{T}} \\
{[\mathrm{mag}]} \\
(9)\end{array}$ & Type & Note \\
\hline 21 & IC 2531 & 095955.7 & -293655 & $1.5 \mathrm{DA}$ & Apr. 0897 & $r$ & 30 & 12.90 & 5.3 & 1 \\
\hline 22 & NGC 3390 & 104804.0 & -313157 & $1.5 \mathrm{DA}$ & Jun. 0298 & $r$ & 30 & 12.85 & 3.0 & 1 \\
\hline 23 & ESO 319-G26 & 113020.0 & -410357 & $2.2 \mathrm{ESO}$ & June 87 & $r$ & & 14.58 & 5.3 & 2 \\
\hline 24 & NGC 3957 & 115401.1 & -193406 & $1.5 \mathrm{DA}$ & Jun. 0398 & $r$ & 15 & 12.81 & -1.0 & 1 \\
\hline \multirow[t]{2}{*}{25} & NGC 4013 & 115831.7 & +435648 & $1.1 \mathrm{LO}$ & Jun. 0197 & $R$ & 18 & 12.19 & 3.0 & 1 \\
\hline & & & & $2.2 \mathrm{CA}$ & Feb. 1598 & $K^{\prime}$ & 33 & - & 3.0 & 1 \\
\hline 26 & ESO 572-G44 & 120109.0 & -202918 & $1.5 \mathrm{DA}$ & Jun. 0398 & $r$ & 20 & 15.04 & 3.0 & 1 \\
\hline 27 & UGC 7170 & 121037.0 & +184924 & $1.1 \mathrm{LO}$ & Mar. 1796 & $R$ & 15 & 14.96 & 6.0 & 1 \\
\hline 28 & ESO 321-G10 & 121142.0 & -383253 & $2.2 \mathrm{ESO}$ & June 87 & $r$ & & 14.22 & 1.4 & 2 \\
\hline 29 & NGC 4217 & 121550.9 & +470532 & $1.1 \mathrm{LO}$ & Jun. 0197 & $R$ & 30 & 12.04 & 3.0 & 1 \\
\hline 30 & NGC 4244 & 121730.0 & +374827 & $1.1 \mathrm{LO}$ & Jun. 0197 & $R$ & 20 & 10.88 & 6.0 & 1 \\
\hline \multirow{2}{*}{31} & UGC 7321 & 121734.1 & +223221 & $1.1 \mathrm{LO}$ & Mar. 1696 & $R$ & 20 & 14.15 & 7.0 & 1 \\
\hline & & & & $1.2 \mathrm{CA}$ & Mar. 0296 & $H$ & 59 & - & 7.0 & 1 \\
\hline \multirow[t]{2}{*}{32} & NGC 4302 & 122142.5 & +143605 & $1.0 \mathrm{HL}$ & May 1996 & $R$ & 10 & 12.50 & 5.0 & 1 \\
\hline & & & & $1.2 \mathrm{CA}$ & Mar. 0296 & $H$ & 53 & - & 5.0 & 1 \\
\hline \multirow[t]{2}{*}{33} & NGC 4330 & 122316.5 & +112206 & $1.5 \mathrm{DA}$ & Apr. 0997 & $r$ & 40 & 13.09 & 6.0 & 1 \\
\hline & & & & $2.2 \mathrm{ESO}$ & Apr. 1097 & $K^{\prime}$ & 32 & - & 6.0 & 1 \\
\hline \multirow[t]{2}{*}{34} & NGC 4565 & 123620.6 & +255905 & $1.0 \mathrm{HL}$ & May 2096 & $R$ & 10 & 10.42 & 3.0 & 1 \\
\hline & & & & $2.2 \mathrm{CA}$ & Feb. 1698 & $K^{\prime}$ & 13 & - & 3.0 & 1 \\
\hline \multirow[t]{2}{*}{35} & NGC 4710 & 124939.0 & +150955 & $1.1 \mathrm{LO}$ & Jun. 0397 & $R$ & 15 & 11.91 & -1.0 & 1 \\
\hline & & & & $2.2 \mathrm{CA}$ & Feb. 1698 & $K^{\prime}$ & 20 & - & -1.0 & 1 \\
\hline \multirow[t]{3}{*}{36} & NGC 5170 & 132949.0 & -175759 & $1.5 \mathrm{DA}$ & Apr. 0897 & $r$ & 45 & 12.06 & 5.0 & 1 \\
\hline & & & & $1.2 \mathrm{CA}$ & Mar. 0396 & $H$ & 11 & - & 5.0 & 1 \\
\hline & & & & $1.2 \mathrm{CA}$ & Mar. 0596 & $K$ & 8 & - & 5.0 & 1 \\
\hline 37 & ESO 510-G18 & 135532.0 & -272447 & $1.5 \mathrm{DA}$ & Jun. 0398 & $r$ & 30 & 16.21 & 1.0 & 1 \\
\hline \multirow{2}{*}{38} & UGC 9242 & 142520.9 & +393222 & 1.1 LO & Mar. 1596 & $R$ & 21 & 14.09 & 7.0 & 1 \\
\hline & & & & $1.2 \mathrm{CA}$ & Mar. 0496 & $H$ & 60 & - & 7.0 & 1 \\
\hline 39 & NGC 5775 & 145357.7 & +033240 & $1.5 \mathrm{DA}$ & Jun. 0398 & $r$ & 15 & 12.24 & 5.0 & 1 \\
\hline \multirow[t]{2}{*}{40} & NGC 5907 & 151553.8 & +561946 & $1.2 \mathrm{CA}$ & Jun. 0296 & $R$ & 30 & 11.12 & 5.0 & 1 \\
\hline & & & & $1.2 \mathrm{CA}$ & Jun. 0496 & $H$ & 6 & - & 5.0 & 1 \\
\hline \multirow[t]{2}{*}{41} & NGC 5908 & 151643.5 & +552440 & $1.2 \mathrm{CA}$ & Sep. 0896 & $R$ & 45 & 12.79 & 3.0 & 1 \\
\hline & & & & $2.2 \mathrm{CA}$ & Feb. 1698 & $K^{\prime}$ & 33 & - & 3.0 & 1 \\
\hline 42 & ESO 583-G08 & 155750.5 & -222947 & $1.5 \mathrm{DA}$ & Jun. 0498 & $r$ & 35 & - & 4.0 & 1 \\
\hline 43 & NGC 6181 & 163220.9 & +194930 & $1.1 \mathrm{LO}$ & Jun. 0397 & $R$ & 15 & 12.49 & 5.0 & 1 \\
\hline 44 & ESO 230-G11 & 184624.0 & -520923 & $1.5 \mathrm{DA}$ & Jun. 0498 & $r$ & 30 & 13.74 & 4.0 & 1 \\
\hline 45 & NGC 6722 & 190340.0 & -645341 & $2.2 \mathrm{ESO}$ & June 87 & $r$ & & 13.54 & 3.0 & 2 \\
\hline 46 & ESO 461-G06 & 195155.9 & -315852 & $1.5 \mathrm{DA}$ & Jun. 0498 & $r$ & 40 & 16.21 & 5.0 & 1 \\
\hline 47 & ESO 339-G16 & 200007.0 & -404303 & $2.2 \mathrm{ESO}$ & June 87 & $r$ & & 16.50 & 1.0 & 2 \\
\hline 48 & IC 4937 & 200518.0 & -561520 & $2.2 \mathrm{ESO}$ & June 87 & $r$ & & 14.86 & 3.0 & 2 \\
\hline 49 & ESO 187-G08 & 204325.2 & -561217 & $1.5 \mathrm{DA}$ & Jun. 0498 & $r$ & 30 & 15.69 & 6.0 & 1 \\
\hline \multirow[t]{2}{*}{50} & IC 5052 & 205206.3 & -691214 & $1.5 \mathrm{DA}$ & Jun. 0398 & $r$ & 35 & 11.16 & 7.0 & 1 \\
\hline & & & & $2.2 \mathrm{ESO}$ & Apr. 1097 & $K^{\prime}$ & 31 & - & 7.0 & 1 \\
\hline 51 & IC 5096 & 211822.0 & -634541 & $1.5 \mathrm{DA}$ & Jun. 0398 & $r$ & 30 & 13.30 & 4.0 & 1 \\
\hline 52 & ESO 466-G01 & 214232.0 & -292210 & $2.2 \mathrm{ESO}$ & June 87 & $r$ & & 14.63 & 2.0 & 2 \\
\hline 53 & ESO 189-G12 & 215538.7 & -545233 & $1.5 \mathrm{DA}$ & Jun. 0498 & $r$ & 30 & 15.59 & 5.0 & 1 \\
\hline 54 & UGC 11859 & 215807.3 & +010034 & $2.2 \mathrm{ESO}$ & June 87 & $r$ & & 15.16 & 4.0 & 2 \\
\hline 55 & ESO 533-G04 & 221403.2 & -265618 & $1.5 \mathrm{DA}$ & Jun. 0398 & $r$ & 70 & 14.18 & 4.8 & 1 \\
\hline 56 & IC 5199 & 221933.0 & -373201 & $1.5 \mathrm{DA}$ & Jun. 0498 & $r$ & 30 & 15.00 & 3.0 & 1 \\
\hline 57 & UGC 11994 & 222053.4 & +331734 & $1.0 \mathrm{HL}$ & Sep. 0797 & $R$ & 54 & 14.85 & 4.0 & 1 \\
\hline \multirow[t]{2}{*}{58} & UGC 12281 & 225912.4 & +133621 & $1.2 \mathrm{CA}$ & Sep. 0796 & $R$ & 60 & 14.79 & 8.0 & 1 \\
\hline & & & & $2.2 \mathrm{CA}$ & Sep. 0496 & $K$ & 20 & - & 8.0 & 1 \\
\hline \multirow[t]{2}{*}{59} & UGC 12423 & 231306.0 & +062400 & $1.2 \mathrm{CA}$ & Sep. 0796 & $R$ & 60 & 14.53 & 5.0 & 1 \\
\hline & & & & $2.2 \mathrm{CA}$ & Sep. 0396 & $K$ & 27 & - & 5.0 & 1 \\
\hline 60 & NGC 7518 & 231312.9 & +061916 & $1.1 \mathrm{LO}$ & Jun. 0297 & $R$ & 40 & 14.24 & 1.0 & 1 \\
\hline 61 & ESO 604-G06 & 231454.0 & -205944 & $1.5 \mathrm{DA}$ & Jun. 0398 & $r$ & 40 & 15.00 & 4.0 & 1 \\
\hline
\end{tabular}

a) $\mathrm{nf}=$ no filter used. 